\title{
Late Cerebellar Vermis Metastasis of Breast Cancer Presenting as Pseudo- Benign Paroxysmal Positional Vertigo
}

\section{Comacchio $\mathrm{F}^{1}$, Mion $\mathrm{M}^{* 2}$ and Markova $\mathrm{V}^{2}$}

${ }^{1}$ Regional Specialized Vertigo Center, Institute of Otolaringology, Department of Neurosciences, Padova University, Padova, Italy

${ }^{2}$ Institute of Otolaringology, Department of Neurosciences, Padova University, Padova, Italy

${ }^{*}$ Corresponding author: Mion M, M.D., Institute of Otolaryngology, Department of Neurosciences, Padova University, Via Giustiniani 2, 35121 Padova, Italy, Fax: +39 049 8213113, Tel: +39 049 8218626, E-mail: med. mion@gmail.com

Citation: Comacchio F, Mion M, Markova V (2016) Late Cerebellar Vermis Metastasis of Breast Cancer Presenting as Pseudo-Benign Paroxysmal Positional Vertigo. J Case Rep Stud 4(6): 604. doi: 10.15744/23489820.4.604

Received Date: November 09, 2016 Accepted Date: December 27, 2016 Published Date: December 29, 2016

\begin{abstract}
Benign paroxysmal positional vertigo (BPPV) is a common peripheral vestibular disease; however intracranial lesions can mimic it. Intractable, not self-limiting paroxysmal positional vertigo sustained by intracranial tumors is called malignant paroxysmal positional vertigo (MPPV) while, when radiological imaging shows vascular cerebellar vermis lesions and there are atypical findings on the DixHallpike maneuver, we speak about pseudo-benign paroxysmal type (pseudo-BPPV).

We present the case of a 53-years-old woman who presented a pseudo-BPPV as the first sign of a cerebellar vermis late metastasis from a breast cancer treated 13 years before.

Lesions of the malignancy located in cerebellar vermis provoked a MPPV, but the nature of the pathology is different from that of pseudo-BPPV, which predominantly has vascular origin. However, there may be forms of pseudo-BPPV caused by malignancies, as in our case.

As regard the differentiation between pseudo-BPPV and MPPV, a radiological investigation must be undertaken in every doubtful case.

Keywords: Cerebellar; Metastasis; Nystagmus; Paroxysmal; Pseudo-Benign Vertigo
\end{abstract}

\section{Introduction}

Benign paroxysmal positional vertigo (BPPV) has been one of the most commonly diagnosed vertigo. It has generally been regarded as due to the displacement of otoconia from the utricle to one or more of the semicircular canals, where they either adhere to the cupula or remain free to gravitate to the momentarily lowest point of the canal. A relevant percentage of paroxysmal positional vertigo appears to be intractable despite canalith repositioning procedure (CRP). This type of positional vertigo is sometime sustained by the action of intracranial tumors that mimic the clinical aspects of BPPV. According to Sakata et al. [1], these cases are considered as malignant paroxysmal positional vertigo (MPPV).

Sakata et al. [2] published a report in 1987 of 20 patients with a presentation of positional nystagmus of benign paroxysmal type, and a follow up study in 1991 of 27 more cases, who were found to have cerebellar vermis lesions on computed tomography (CT) scan (pseudo-benign paroxysmal type, pseudo-BPPN). In particular, they saw evidence of cerebellar vermis infarction in the vast majority of cases. Their conclusion was that there is a causal role for vascular cerebellar vermis lesions. Büttner et al. have classified this kind of feature as central PPV [3]. Lesions in central paroxysmal positional vertigo are often dorsolateral to the fourth ventricle or in the dorsal vermis [4]. Barber [5] published a case of vermis metastasis in a patient presenting with features of postural vertigo and paroxysmal down beat positional nystagmus, and Watson and Terbrugge [6] reported BPPN as first sign of cerebellar medulloblastoma.

We describe the case of a patient who was initially misdiagnosed as having BPPV, with subsequent delay in the diagnosis of an underlying cerebellar vermis late metastasis from a breast cancer treated 13 years before.

\section{Case Report}

A 53-years old female was admitted to Emergency Room with two days' history of subjective vertigo lasting seconds, triggered by 
head movements and worsened by left lateral decubitus, associated with instability of the march, nausea and vomiting. She didn't complain of other cochlear or neurological symptoms. In history she reported right mastectomy and chemotherapy performed 13 years ago for right breast cancer, which histological evidenced of "mammary multicentric infiltrative carcinoma with infiltrative ductal aspects (G2 according to Elston-Ellis classification), associated with infiltrative lobular carcinoma and intraductal carcinoma with solid and comedonic features (pT1 cN1 biMx)".

On ENT bed-side examination performed in emergency, otoscopy was normal; spontaneous nystagmus wasn't apparent; Romberg test and Barany test were within normal limits; Unterberger test showed a slight deviation to the left; positional and positioning manoeuvres appeared unremarkable. The neurological examination confirmed the findings of ENT evaluation. Brain CT scan resulted negative. Since the patient reported a symptoms' improvement after few hours, she was discharged from the Emergency Room. Three days later, she was admitted in our Unit for recurrence of vertigo for further investigation. Intracranic and supraaortic vessels echo colordoppler resulted normal. Audiological assessment was in the normal limits.

Videonystagmography showed absence of spontaneous nystagmus. Oculomotricity parameters were normal. A rotatory left torsional paroxysmal positioning nystagmus was evoked with left Dix-Hallpike manoeuvre. The nystagmus presented a latency of $5 \mathrm{sec}$., a $20 \mathrm{sec}$. of duration, but with scarce amplitude and lacking of a crescendo-decrescendo pattern (Figure 1). The nystagmus presented as not fatigable, and attempts of liberation with Semont manoeuvre were unsuccessful. For these reasons a cerebral and brain stem MRI with gadolinium and angio-MRI was prescribed. The imaging evidenced signal's abnormalities of culmen region in subtentorial site, with contrast impregnation extending to the adjacent folia (Figure 2a,b and c).
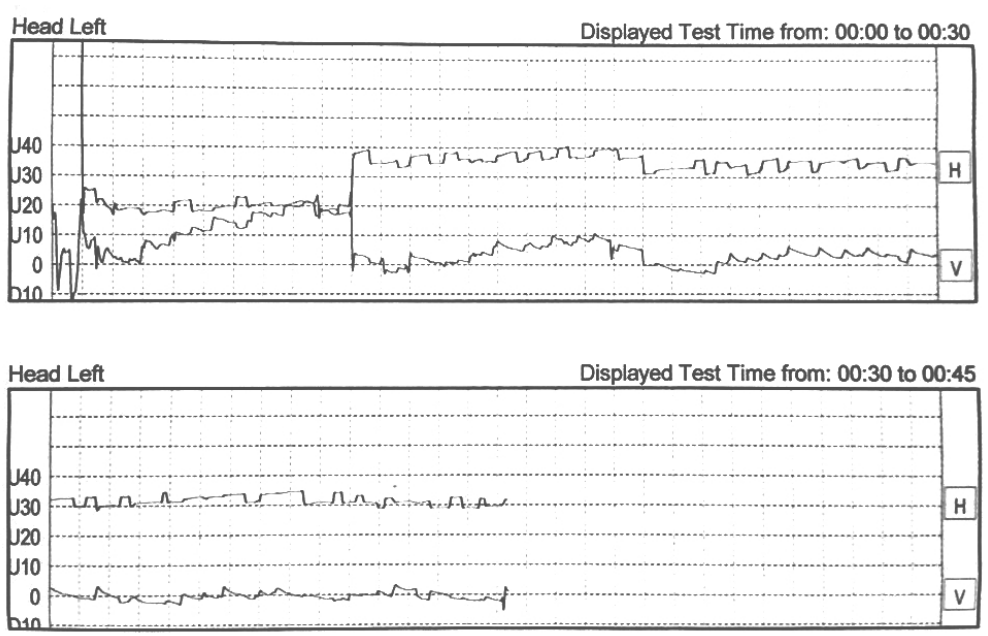

Figure 1: Videonystagmography: left Dix-Hallpike manoeuvre

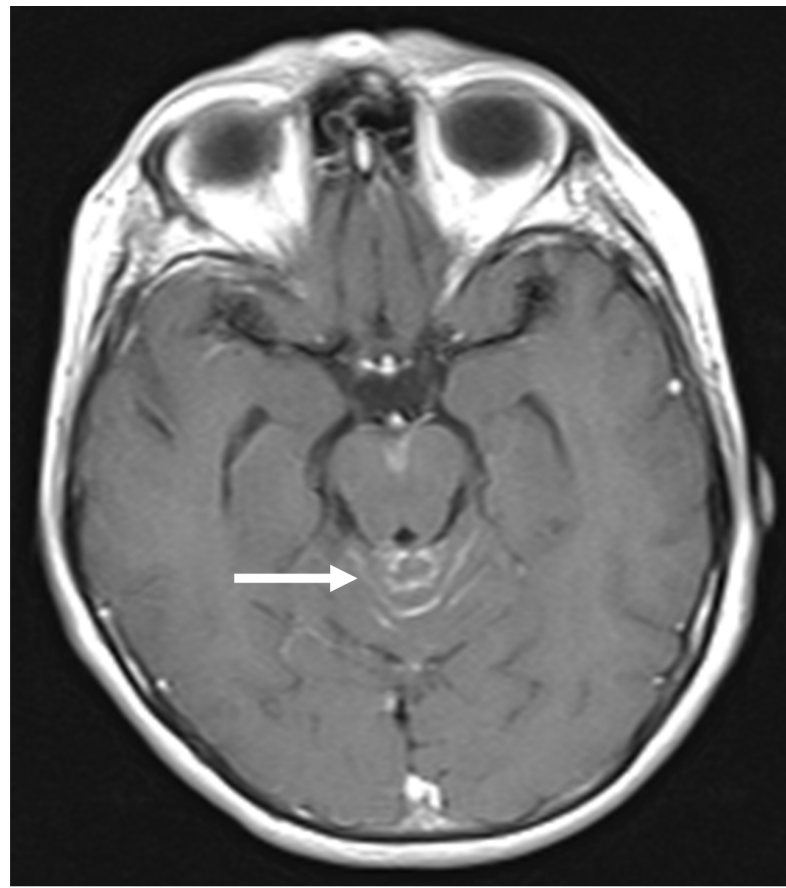

Figure 2A: Cerebral and brain stem MRI with gadolinium: axial view 


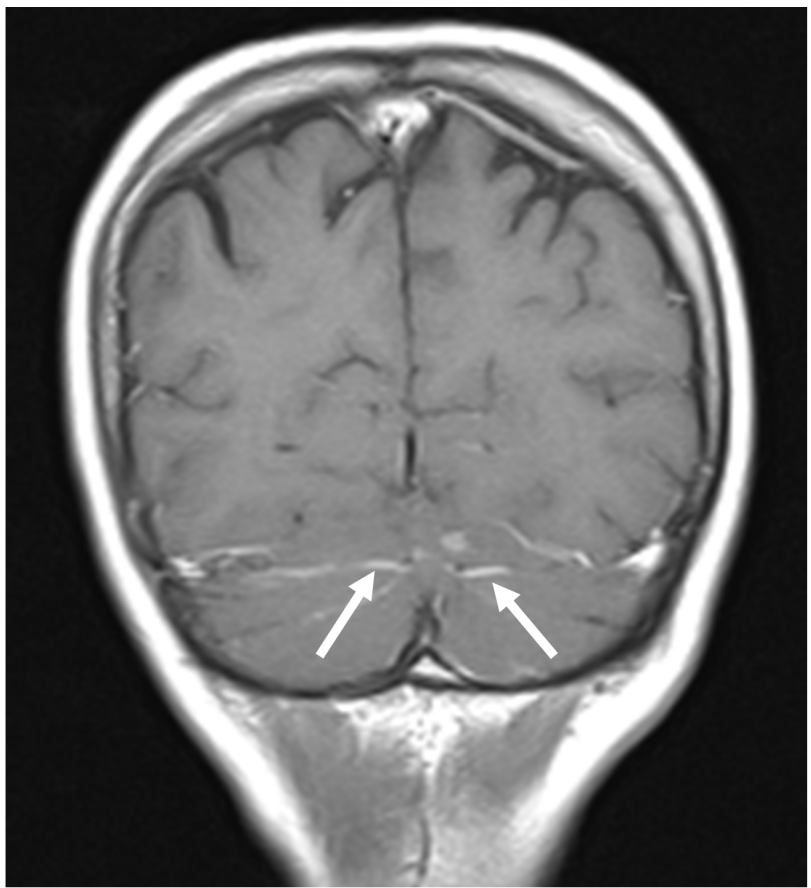

Figure 2B: Cerebral and brain stem MRI with gadolinium: coronal view

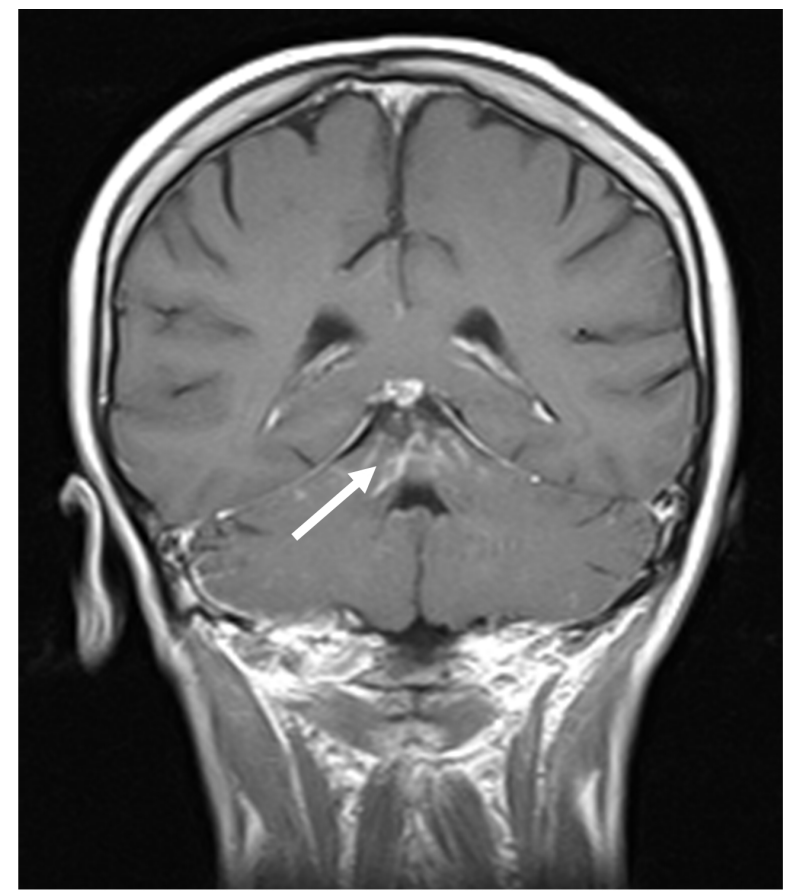

Figure 2C: Cerebral and brain stem MRI with gadolinium: coronal view

Spinal tap was compatible with localization of mammary carcinoma: histological examination of the cerebrospinal fluid showed numerous groups of cells with varying sizes, from medium-large to very large, with wide cytoplasm and hyperchromic nucleus, mixed with small to medium size lymphomonocitoid elements. These aspects were indicative of meningeal carcinomatosis. Several blood tumor markers were altered (ferritin, CEA, Ca 19-9, Ca 125, Ca 15-3). She was evaluated in the Oncologic Unit for eventual palliative treatments. She died few months later for bronchopneumonia developed after cerebral edema.

\section{Discussion}

BPPV is an extremely common cause of vertigo, with a benign natural history. It might be difficult to distinguish between central and peripheral origin of paroxysmal nystagmus if we consider some features in detail, i.e. 1) the latency of onset of symptoms after diagnostic maneuvers, 2) the duration of nystagmus, 3) the course of the nystagmus (with a crescendo - decrescendo pattern for the peripheral cases), 4) vertigo. Upbeat, downbeat and pure torsional nystagmus typically indicates a central origin, as well as the absence of vertigo with positional or positioning nystagmus [3] (Table 1). 


\begin{tabular}{|c|c|}
\hline Positional Peripheral Nystagmus & Central Nystagmus \\
\hline $\begin{array}{c}\text { Delayed onset with movements } \\
(5-40 \mathrm{sec})\end{array}$ & Without latency \\
\hline Disappears after sec - min & Persistent \\
\hline Intense vertigo & Possible soft or absent vertigo \\
\hline Relieved by gaze fixation & Persistent \\
\hline Fatigable & Not fatigable \\
\hline $\begin{array}{c}\text { Paroxysmal with crescendo- } \\
\text { decrescendo pattern }\end{array}$ & $\begin{array}{c}\text { Paroxysmal without crescendo- } \\
\text { decrescendo pattern }\end{array}$ \\
\hline
\end{tabular}

Table 1: Positional Peripheral Vs Central Nystagmus

Often the direction of nystagmus during an attack can allow differentiation between peripheral and central origin [4,7] but this concept cannot be applied to all the cases: although BPPV of posterior canal origin and anterior canal origin both give rise to geotropic rotatory beating, in both cases the observed nystagmus also has a vertical component. Furthermore, multiple positional nystagmus can suggest multiple canal involvement in BPPV [8].

Positional nystagmus in intracranial tumors was already noted in 1950 by Nylen [9], who described a "positional changing" nystagmus associated with posterior fossa tumors. These expansive intracranial processes could generate a positional vertigo [10], but they are not intrinsic to labyrinth and they are not benign or self-limiting diseases. Sakata et al. [1] presented a malignant type of paroxysmal positional vertigo (MPPV) [1], which is related to lesions of the central nervous system.

De Stefano et al. [11] describe four main properties that may suggest a MPPV and they all emerge from an accurate otoneurological evaluation: 1) Modification of the nystagmus characteristics after several diagnostic maneuvers done to evaluate BPPV. 2) Presence of associated symptoms that are uncommon in BPPV: persisting disequilibrium, headache, hemifacial paresthesia or facial nerve palsy, cochlear symptoms. 3) Absence of previous history of BPPV resolved by CRP. 4) Failure of several canalith repositioning maneuvers and persistence of symptoms and signs for more than one month. An example of association of BPPV and cerebellar lesion in which the symptoms completely disappeared after CRP was described by Lynn and Brey [12].

Lesions of the malignant type can be located in cerebellar vermis, but the nature of the pathology is different from that of pseudoBPPN, which is predominantly vascular according to studies of Sakata et al. [2].

Two cerebellar locations can be related to the occurrence of pseudo-BPPN: one involves the nodulus/uvula region, the other location is in the vicinity of, but clearly separate from, the nodulus/uvula region dorsolateral to the fourth ventricle [13,14]. The mechanism hypothesized at the basis may be explained by incomplete inhibition of the vestibule-oculomotor system including the cerebellar flocculonodular lobe or vestibule-cerebellum [2].

The symptoms of pseudo-BPPN are characterized by closely resembled to that of BPPV. It's a pure rotatory counter-rolling positional or positioning nystagmus with a latent period, with less fatigability of the provoked vertiginous attacks [2]. According to Sakata et al. [2], the counter-rolling nystagmus, best elicited by the positioning nystagmus test, can't be a definite diagnostic sign for the BPPN, since those type of nystagmus could also be found in cerebellar vermis lesion (pseudo-BPPN). The only definite difference between pseudo-BPPN and genuine posterior canal BPPN in canalolithiasis is the constant appearance of the nystagmus in repeated examination [2].

On the other hand, the difference between our report and the nystagmus in a cupololithiasis of posterior canal is that the latter is long-lasting, and it reverses direction in nose-down position.

So, atypical physical findings on the Dix-Hallpike maneuver must be considered a red flag [15] (in our case, the absence of a crescendo - decrescendo pattern of the nystagmus after positioning maneuver).

Our report shows the peculiar case of a pseudo-BPPV not due to a vascular lesion, but provoked by a malignant disease, which represents a second distinctive tract, since it's the first sign of late cerebellar vermis metastasis from breast cancer. Another similar case was reported by Watson and Terbrugge, describing a positional nystagmus of benign paroxysmal type with posterior fossa medulloblastoma [6]. However, in their case, the indication of a neuroradiologic assessment was suggested by a history of eight months of headache. In our case the lacking of crescendo-decrescendo pattern was the distinctive tract of the nystagmus suspected for central origin.

The median latency between breast cancer diagnosis and brain metastasis from breast cancer is 34 months [16]. We define "late metastasis" as those metastases that appear at least 10 years later after breast cancer diagnosis [16]. This is a rare event, however if a patient's clinical history reports a surgical treatment for breast cancer, even if more than 10 years before, in the presence of specific neurologic symptoms and neuroradiologic evidence of a cerebral (or cerebellar) neoplasm, we should always keep in mind that it might be a late brain metastasis of breast cancer [16]. 


\section{Conclusion}

Pseudo-BPPN can usually be identified after a full examination with brain imaging, since those type of nystagmus could be found in vascular cerebellar vermis lesion. Lesions of the malignant type are also located in cerebellar vermis, but the nature of the pathology is different from that of pseudo-BPPN: MPPV is a positional paroxysmal and not self-limiting vertigo supported by an intracranial tumor interacting with the vestibular pathways. We describe the rare case of pseudo-BPPV as first sign of late cerebellar vermis metastasis from breast cancer. It's describe the possibility that pseudo-BPPV could be supported by a malignant lesion, and not also by vascular infarctions of cerebellar vermis.

\section{References}

1. Sakata E, Uchida Y, Nakano Y, Takahashi K (1984) Pathophysiology of positional vertigo of the malignant paroxysmal type. Auris Nasus Larynx 11: 79-90.

2. Sakata E, Ohtsu K, Itoh Y (1991) Positional nystagmus of benign paroxysmal type (BPPN) due to cerebellar vermis lesions: Pseudo-BPPN. Acta Otolaryngol Suppl 481: 254-7.

3. Büttner U, Helmchen C, Brandt T (1999) Diagnostic criteria for central versus peripheral positioning nystagmus and vertigo: a review. Acta Otolaryngol 119: $1-5$.

4. Shoman N, Longridge N (2007) Cerebellar vermis lesions and tumours of the fourth ventricle in patients with positional and positioning vertigo and nystagmus. J Laryngol Otol 121: 166-9.

5. Barber HO (1984) Positional nystagmus. Otolaryngol Head Neck Surg 92: 649-55.

6. Watson CP, Terbrugge K (1982) Positional nystagmus of the benign paroxysmal type with posterior fossa medulloblastoma. Arch Neurol 39: 601-2.

7. Jacobson GP, Butcher JA, Newman CW, Monsell EM (1995) When paroxysmal positioning vertigo isn’t benign. J Am Acad Audiol 6: 346-9.

8. Lopez-Escamez JA, Molina MI, Gamiz M, Fernandez-Perez AJ, Gomez M, et al. (2005) Multiple positional nystagmus suggests multiple canal involvement in benign paroxysmal vertigo. Acta Otolaryngol 125: 954-61.

9. Nylen CO (1950) Positional nystagmus; a review and future prospects. J Laryngol Otol 64: 295-318.

10. De Stefano A, Dispenza F, Di Trapani G, Kulamarva G (2008) Meningioma of the cerebellopontine angle mimicking benign paroxysmal positional vertigo. J Otolaryngol Head Neck Surg 37: E46-8.

11. De Stefano A, Kulamarva G, Dispenza F (2012) Malignant paroxysmal positional vertigo. Auris Nasus Larynx 39: 378-82.

12. Lynn S, Brey R (1993) Benign paroxysmal positioning vertigo with indeterminate cerebellar lesion: case report. J Am Acad Audiol 4: 384-91.

13. Noh KJ, Park SC, Kim HJ, Choi HS (2012) A Case of Cerebellar Hemangioblastoma Mimicking Benign Paroxysmal Positional Vertigo. Korean JOtorhinolaryngolHead Neck Surg 55: 58-61.

14. Hosomi M, Matsumoto K, Kageyama Y, Ichikawa K (2008) Three cases of small brain infarction in the lateral wall of the fourth ventricle presenting with paroxysmal positional vertigo and vomiting [in Japanese]. Rinsho Shinkeigaku 48: 709-12.

15. Dunniway HM, Welling DB (1998) Intracranial tumors mimicking benign paroxysmal positional vertigo. Otolaryngol Head Neck Surg 118: 429-36.

16. Piccirilli M, Sassun TE, Brogna C, Giangaspero F, Salvati M (2007) Late brain metastases from breast cancer: clinical remarks on 11 patients and review of the literature. Tumori 93: 150-4. 